\title{
Glicanos de la vellosidad trofoblástica en la anemia ferropénica y la preeclampsia grave
}

\author{
Glycans in villus trophoblast in iron \\ deficiency anemia and early-onset \\ severe preeclampsia
}

\begin{abstract}
Glycoproteins attached to cell membrane of syncytiotrophoblast are in close contact with maternal blood, thus these molecules could participate in cell-to-cell communication and biological functions involving ligand-receptors in the maternal-fetal interphase. The attached glycans are involved in the stability, folding and exportation of the protein towards the cell membrane. The objective of this study was to characterize the glycan profile of third trimester placental villi obtained from pregnant women with early-onset severe preeclampsia and gestational anemia compared with normal pregnant women. Protein extracts from placental villi were used in lectin blot assays. -2,3 N-and O-linked sialic acid was over-expressed in villous of severe preeclamptic placentas measured by MAA lectin staining. High mannose glycans and Gal-GlcNAc patterns were also increased in severe preeclampsia compared with the other groups. These findings can explain changes in the cell membrane expression of glycoproteins.

Key words: Anemia, early-onset severe preeclampsia, glycosylation, Transferrin-receptor 1 .
\end{abstract}

Alejandra María Gómez-Gutiérrez (1) Beatriz Elena Parra-Sosa (2) Julio Cesar Bueno-Sánchez (3)

(1) Grupo Reproducción. Escuela de Nutrición y Dietética. Universidad de Antioquia, Colombia. (2) Grupo de Investigación Alimentación y Nutrición Humana (GIANH-END-U de A). Escuela de Nutrición y Dietética. Universidad de Antioquia. Colombia (3) Grupo Reproducción. Facultad de Medicina. Universidad de Antioquia. Colombia.

Dirigir la correspondencia a: Profesora Alejandra María Gómez Gutiérrez Carrera 53 \#61-30 Laboratorio 534

Medellín-Colombia Tel: 5742196476

E-mail: alejandram.gomezg@gmail.com

Este trabajo fue recibido el 12 de Septiembre de 2014 y aceptado para ser publicado el 10 de Abril de 2015.

\footnotetext{
Comunicaciones previas parciales de este trabajo fueron: Publicadas en modalidad abstract:

Glycosylation patterns of villous placentas in severe preeclampsia and gestational anemia and the relationship with the maternal iron status. Placenta 34 (2013) A1-A94. Presentadas en modalidad poster:

V Latin American Symposium on Maternal Fetal Interaction \& Placenta IV Latin American Symposium on Reproductive Immunology. Foz do Iguaçu, Brasil. Febrero 2013. LIII Congreso Internacional de la Sociedad Mexicana de Nutrición y Endocrinología. Cancún, Mexico. Noviembre 2013.
}

\section{INTRODUCCIÓN}

El estado nutricional materno antes y durante la gestación tiene un gran impacto en los resultados materno-fetales (1). El hierro es un nutriente esencial, cofactor de diferentes enzimas del metabolismo (2), necesario para la síntesis de neurotransmisores y la mielinización del sistema nervioso (3) y un componente básico de la hemoglobina y la mioglobina (2).

Las alteraciones en el estado de hierro materno durante la gestación se han asociado a enfermedades desde la anemia ferropénica hasta la preeclampsia. La anemia, diagnosticada por una reducción en la concentración de hemoglobina materna $(<11 \mathrm{~g} / \mathrm{dl})$ y de la ferritina sérica $(<15 \mathrm{ug} / \mathrm{L})(4)$, disminuye la capacidad de trabajo en la madre, aumenta el riesgo de infecciones y el tiempo de tratamiento (5). En el feto, la anemia se ha asociado con restricción de crecimiento intrauterino (RCIU), bajo peso al nacer $(B P N)(2,6,7)$ y parto pretérmino $(8)$.

La Organización Mundial de la Salud reporta 41,8\% de las gestantes en el mundo con anemia (4) y en Colombia $10.3 \%$ de las embarazadas presentan anemia ferropénica (9). En la región noroccidental de Colombia, conocida como Antioquia, un estudio encontró $50 \%$ de las gestantes en el segundo trimestre con depósitos de hierro insuficientes (ferritina sérica $<24 \mathrm{ng} / \mathrm{mL}$ ) y en el tercer trimestre estuvieron cercanas a la deficiencia de hierro (ferritina sérica $<15 \mathrm{ng} / \mathrm{mL}$ ) (10).

Por otra parte, los desórdenes hipertensivos del embarazo se han asociado también con RCIU, BPN (11) y con fallas en la expansión del volumen plasmático que a su vez conducen a alteraciones en la perfusión utero-placentaria (12). La preeclampsia es la enfermedad más frecuente dentro de los desórdenes hipertensivos en la gestación y se caracteriza por 
hipertensión y alteraciones renales o de otros órganos blanco (13). Si bien se considera una enfermedad multisistémica, su origen se ha asociado a una disminución en la invasión del trofoblasto a las arterias espirales, evento crucial para la transformación histológica de estas arterias a vasos de mayor diámetro y menor presión. Al comprometerse la invasión trofoblástica durante la enfermedad, el aporte de sangre y nutrientes resulta insuficiente para la placenta en crecimiento (11). Bajo esta condición también se ha reportado una alteración en el estado de hierro materno (14-16), con reservas elevadas de hierro durante la gestación ( $>41 \mathrm{ug} / \mathrm{L}$ de ferritina), que se han asociado con parto pretérmino y con las alteraciones de la perfusión arriba descritas. La preeclampsia se ha reportado en $5 \%$ a $8 \%$ de las gestaciones en el mundo (17), y la preeclampsia grave alrededor de 0,59\% (18). En Colombia el trastorno hipertensivo asociado a la gestación es la principal causa de muerte materna (19).

La placenta es una estructura específica del embarazo (20), que emerge de la interacción entre los tejidos embrionarios y maternos (21) y que entre muchas otras funciones se encarga de la nutrición fetal. La vellosidad trofoblástica flotante es la estructura placentaria que cumple con las condiciones para hacer el intercambio materno fetal (22); esta se origina en etapas tempranas de la gestación a partir de las células progenitoras de citotrofoblasto (CTB), las cuales presentan un fenómeno de fusión celular, dando lugar al sincitiotrofoblasto (STB), que se encuentra en contacto directo con la sangre materna (23). El STB transfiere los nutrientes al feto y para ello exhibe en la membrana celular apical, una importante variedad de mecanismos transportadores $(24,25)$; uno de ellos, la proteína relacionada con la captación del hierro materno, el Receptor 1 de Transferrina (TfR1). Estudios iniciales en modelos in vitro e in vivo, determinaron que la expresión del TfR1 aumenta en estados de deficiencia de hierro (26-28), mientras que en la preeclampsia se encontró una expresión reducida, situación que podría contribuir a la RCIU que se presenta en la mayoría de estas gestaciones $(29,30)$.

Es posible que la alteración en la expresión del TfR1 se deba a modificaciones postraduccionales asociadas a la glicosi- lación que pueden tener un papel relevante en la exportación de esta glicoproteína a la membrana del STB; análisis de los glicanos del TfR1 han mostrado que una falla en la glicosilación del TfR1 decrece su migración a la superficie celular, esto posiblemente porque los $\mathrm{N}$-glicanos parecen ser fundamentales para el adecuado plegamiento y para la exportación de la proteína a la membrana celular (31). Además, los glicanos de la membrana de los diferentes tipos de trofoblasto tienen un papel central en procesos de comunicación materno-fetal (señalización, reconocimiento célula-célula y célula-matriz extracelular), de manera que modulan procesos de diferenciación celular, adhesión, implantación (32), proliferación, modulación del sistema inmune, secreción (33) y transporte de nutrientes (34).

El objetivo del presente estudio fue determinar el patrón de glicosilación de la vellosidad placentaria de mujeres con preeclampsia grave y compararlo con el de placentas de mujeres con anemia ferropénica, bajo la hipótesis de que estos estados patológicos que presentan afectación del estado de hierro modifican el perfil de glicosilación de la vellosidad trofoblastica, lo que se reflejaría en cambios del patrón de glicosilación de proteínas particulares como el TfR1.

\section{SUJETOS Y MÉTODOS}

Los procedimientos fueron aprobados por el Comité de Bioética del Instituto de Investigaciones Médicas de la Facultad de Medicina de la Universidad de Antioquia. El estudio fue conducido de acuerdo con los estándares éticos para la experimentación en humanos, establecidos en la declaración de Helsinki y la Resolución 008430 del Ministerio de Salud de Colombia. El consentimiento informado fue firmado por todas las participantes.

El tamaño de muestra se estimó por conveniencia. Las placentas de cada grupo (control C, anemia ferropénica A, preeclampsia grave $P E$ ), se obtuvieron de mujeres sometidas a cesárea electiva, con edades entre 18 y 40 años, con embarazo monofetal, de tercer trimestre e índice de masa corporal gestacional (IMC) normal o en sobrepeso (35). El peso y la estatura se tomaron con equipos de precisión (báscula de

TABLA 1

Criterios de inclusión de las mujeres evaluadas en el estudio

\begin{tabular}{|c|c|c|c|}
\hline Criterio de Inclusión & $\begin{array}{c}\text { Control } \\
n=6\end{array}$ & $\begin{array}{c}\text { Anemia } \\
n=5\end{array}$ & $\begin{array}{l}\text { Preeclampsia } \\
\qquad=6\end{array}$ \\
\hline Edad (años) & $18-40$ & $18-40$ & $18-40$ \\
\hline Tipo de gestación & Mono fetal & Mono fetal & Mono fetal \\
\hline Edad gestacional (semanas) & $30-40$ & $30-40$ & $30-40$ \\
\hline IMC & Normal o Sobrepeso & Normal o Sobrepeso & Normal o Sobrepeso \\
\hline Presión arterial & $<139 / 89$ mmHg & $<139 / 89 \mathrm{mmHg}$ & $>140 / 90 \mathrm{mmHg}$ \\
\hline Proteinuria & No & No & $>300 \mathrm{mg} / 24 \mathrm{~h}$ \\
\hline Hemoglobina & $>11 \mathrm{~g} / \mathrm{dL}$ & $<11 \mathrm{~g} / \mathrm{dL}$ & $>11 \mathrm{~g} / \mathrm{dL}$ \\
\hline Ferritina sérica & $>15 \mathrm{ng} / \mathrm{mL}$ & $<15 \mathrm{ng} / \mathrm{mL}$ & $>15 \mathrm{ng} / \mathrm{mL}$ \\
\hline
\end{tabular}


piso electrónica marca Tanita H327 de 0,1 kg de sensibilidad y capacidad de $150 \mathrm{~kg}$ y estadiómetro Seca de 0,1 cm) y con las técnicas adecuadas para asegurar la calidad de los datos. Los datos de peso y estatura de las mujeres con PE, fueron obtenidos de su historia clínica o del carné prenatal, debido a que la gravedad de las gestantes impedía hacer la toma directa de estos valores (criterios de inclusión en la tabla 1). En las mujeres del grupo con PE se consideraron los siguientes criterios para diagnosticar el cuadro grave de preeclampsia: presión arterial sistólica $>160 \mathrm{mmHg}$ o presión arterial diastólica $>110 \mathrm{mmHg}$ en mujeres sin historia previa de hipertensión, o proteinuria $>$ $5 \mathrm{~g}$ en orina de $24 \mathrm{~h}$, o trombocitopenia (plaquetas $<100.000 /$ $\mathrm{ml}$ ) (36). Se seleccionaron sólo las muestras procedentes de mujeres que a las 12 semanas posparto habían retornado a valores de presión arterial normales, indicando que la gestante desarrolló preeclampsia y no hipertensión crónica.

Se consideraron como criterios de exclusión: la negativa de la paciente, sus familiares o del médico tratante para ingresar al estudio; pacientes con estado nutricional clasificado como bajo peso u obesidad; con enfermedad de base claramente diagnosticada que pudiera alterar el curso clínico de las enfermedades en estudio o que pudiera perturbar los hallazgos esperados, como enfermedad renal, hipertensión crónica, infecciones agudas o crónicas, enfermedades autoinmunes, cáncer o alteraciones del colágeno o el uso de sustancias psicoactivas.

Determinación de hemoglobina, ferritina y proteína $C$ reactiva: En el caso de las muestras de los grupos $C$ y $A$ se tomó muestra de sangre venosa $(9 \mathrm{~mL})$ en tubo tapa roja. La ferritina sérica y la hemoglobina se analizaron por inmunoensayo de electroquimioluminiscencia (ECLIA) en un analizador automático (Roche Modular Analytics E170) y por el método de la cianometahemoglobina modificado, en equipo de lectura automática (CELL DYN 3700 de Abbott), respectivamente. La PCR se cuantificó por inmunoturbidimetría, en un analizador automático (Modular P800 de Roche) y se utilizó para interpretar adecuadamente los resultados de ferritina materna (37). Valores $>1,5 \mathrm{mg} / \mathrm{dL}$, indicativos de inflamación en gestantes, con una concentración de ferritina sérica $<30 \mu \mathrm{g} / \mathrm{L}$, se clasificaron como depleción de hierro y una concentración $\geq 30 \mu \mathrm{g} / \mathrm{L}$ indicó que no existía ferropenia o deficiencia de hierro. En el caso de las mujeres con preeclampsia, el valor de hemoglobina se obtuvo de las historias clínicas. Se tomó además muestra de sangre venosa $(9 \mathrm{~mL})$ previa a la cesárea, en tubo tapa roja, para la determinación de ferritina y PCR, como se describió previamente.

Obtención de vellosidad placentaria: Se aisló vellosidad trofoblástica placentaria de acuerdo con una modificación del método de Kliman (38). De cada placenta se obtuvieron alrededor de 4 cotiledones y se disecaron por disgregación mecánica luego de retirar la placa basal de la decidua. El tejido trofoblástico se lavó con tampón fosfato salino (phosphate buffered saline PBS) suplementado con antibióticos al 1\%. Los fragmentos de vellosidad placentaria se almacenaron a $-70^{\circ} \mathrm{C}$ hasta ser procesados. De cada muestra, $200 \mathrm{mg}$ de vellosidad trofoblástica se homogenizaron mecánicamente, en solución salina $(\mathrm{NaCl} 0.9 \%)$ y en presencia de inhibidor de proteasa (Protease Inhibitor Cocktail Set 1 Calbiochem ${ }^{\circledR}$, AEBSF $100 \mathrm{~m} M$, Aprotinina bovina $80 \mathrm{u} M$, Bestanina $5 \mathrm{~m} M$, E-64 inhibidor $1.5 \mathrm{mM}$ y Leupeptina $2 \mathrm{mM}$ ). La suspensión se centrifugó a $15000 \mathrm{rpm} / 4^{\circ} \mathrm{C} / 15 \mathrm{~min}$ y se tomó el sobrenadante. Se cuantificó la concentración de las proteínas mediante el uso del Kit comercial Pierce ${ }^{\mathrm{TM}}$ BCA Protein Assay.

Identificación de patrones de glicanos en proteínas de la vellosidad placentaria: Se realizó electroforesis en geles de poliacrilamida al 10\% y sodio dodecil sulfato 1\% (SDS-PAGE). Se disolvieron 15 ug de proteínas en tampón de carga (Tris $\mathrm{HCl} 0,5 \mathrm{M} \mathrm{pH} 6,8$, glicerol 30\%, mercaptoetanol 6\%, SDS $10 \%$, azul de bromofenol 0,01\% en agua destilada); se hizo la separación de proteínas a 130V. El gel fue teñido con azul de coomassie para caracterizar los pesos moleculares de las diferentes proteínas. Se realizó la separación de las proteínas por medio del sistema Experion ${ }^{\mathrm{TM}}$ Automated Electrophoresis System-BIORAD. Luego, las proteínas fueron transferidas a una membrana de nitrocelulosa (Whatman, GE Healthcare) por medio de una transferencia semiseca, usando towin con metanol al 20\% v/v, a $116 \mathrm{~mA}$ por cada membrana con un área de $40 \mathrm{~cm}$. Se realizó el Lectin blot haciendo uso del Kit de Diferenciación de Glicanos DIG (ROCHE) (tabla 2). Después del bloqueo se incubó con la lectina acoplada a digoxigenina durante 1 hora. Luego se procedió con la incubación en solución con anticuerpo policlonal de oveja anti-digoxigenina conjugado con fosfatasa alcalina, $0.1 \%$ v/v durante 1 hora. Para la detección del complejo lectina-carbohidrato, las membranas se sumergieron en solución de tinción nitroazul de tetrazolio/ BCIP 5-bromo-cloro-3-indolil fosfato $2.0 \% \mathrm{v} / \mathrm{v}$, preparada en

\section{TABLA 2}

Características de las lectinas del Kit de Diferenciación de Glicanos DIG, ROCHE

\begin{tabular}{|c|c|c|c|c|}
\hline Lectina & Abreviatura & Especificidad de unión & Concentraciones & Control positivo \\
\hline Galanthus nivalis agglutinin & GNA & $\begin{array}{l}\text { Man terminal (1-3), } \\
(1-6) \text { o (1-2) unido a man. }\end{array}$ & $0.1 \% \mathrm{v} / \mathrm{v}$ & Carboxipeptidasa Y \\
\hline Sambucus nigra agglutinin & SNA & $\begin{array}{l}\text { Ácido sialico (2-6) } \\
\text { unido a Gal. }\end{array}$ & $0.1 \% \mathrm{v} / \mathrm{v}$ & $\begin{array}{l}\text { Transferrina y/o } \\
\text { Fetuina }\end{array}$ \\
\hline Maackia amurensis agglutinin & MAA & $\begin{array}{c}\text { Ácido sialico (2-3) } \\
\text { unido a Gal. }\end{array}$ & $0.5 \% \mathrm{v} / \mathrm{v}$ & Fetuina \\
\hline Peanut agglutinin & PNA & $\begin{array}{c}\text { Core disacárido de Gal (1-3) } \\
\text { unido a GalNAc }\end{array}$ & $1.0 \% \mathrm{v} / \mathrm{v}$ & Asialofetuina \\
\hline Datura stramonium agglutinin & DSA & Gal (1-4) unido a GlcNAc & $0.1 \% \mathrm{v} / \mathrm{v}$ & $\begin{array}{l}\text { Fetuina y/o } \\
\text { Asialofetuina }\end{array}$ \\
\hline
\end{tabular}


tampón 2 (0.1 M Tris- $\mathrm{HCl}, 0.05 \mathrm{M} \mathrm{MgCl} 2,0.1 \mathrm{M} \mathrm{NaCl}, \mathrm{pH}$ 9.5) (figura 1).

Análisis estadístico: Se calculó la intensidad y el área de las bandas de glicanos unidos a proteínas por medio del software Image J versión 1.44. Se hizo una densitometría y normalización usando una banda escasamente glicosilada correspondiente a $67 \mathrm{kDa}$ detectada tanto en los Lectin Blot (figura 1), como en la electroforesis capilar. Se compararon de los grupos con la prueba de Kruskal-wallis para datos con una distribución no paramétrica. Los datos se presentaron como medianas y sus rangos. Los análisis se hicieron usando el programa GraphPad PRISM 5.0® (Software Inc., La Jolla, (A, USA).

\section{RESULTADOS}

La tabla 3 resume las características clínicas de los tres grupos de mujeres, las cuales corresponden a los criterios de inclusión definidos para cada uno.

Expresión de proteínas en la vellosidad placentaria: En las diferentes electroforesis realizadas, se encontró una expresión

\section{FIGURA 1}

Imagen representativa de un lectin blot

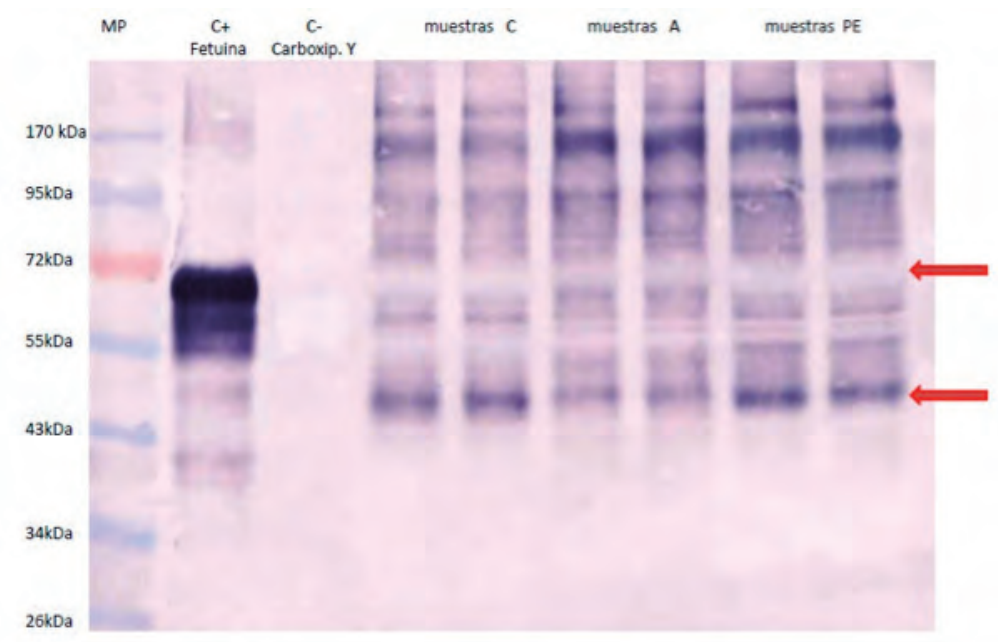

Imagen de un lectin blot usando lectina DSA, en el que se puede ver la expresión diferencial de Gal-GlcNAc determinada a partir de la intensidad en las bandas de glicoproteínas en las muestras que pertenecían a cada uno de los grupos. La flecha superior indica la banda de 67KDa escasamente glicosilada de expresión constante y la flecha inferior indica una de las bandas que fueron analizadas. En cada ensayo se utilizaron proteínas conocidas como control positivo y negativo.

TABLA 3

Características clínicas de las mujeres participantes en el estudio

\begin{tabular}{|c|c|c|c|c|}
\hline $\begin{array}{l}\text { Variable } \\
n=6\end{array}$ & $\begin{array}{l}\text { Control } \\
n=5\end{array}$ & $\begin{array}{c}\text { Anemia } \\
n=6\end{array}$ & $\begin{array}{c}\text { Preeclampsia } \\
\text { estadística }\end{array}$ & Significancia \\
\hline Edad materna (años) & $26,5(20-39)$ & $24(20-24)$ & $26,5(18-35)$ & p 0,0943 \\
\hline Grávidas & $2(1-4)$ & $3(1-3)$ & $1,5(0-3)$ & p 0,3382 \\
\hline Edad gestational (semanas) & $39(37-39) * *$ & $38(37-39)$ & $31,5(30-33)^{* *}$ & p 0,0023 \\
\hline IMC Materno (k/m2) & $28,2(25-30)$ & $26,3(24,6-26,7)$ & $27,9(24-30,4)$ & p 0,1802 \\
\hline Hemoglobina materna $(\mathrm{g} / \mathrm{dL})$ & $13,4(11,9-15,1)^{* *}$ & $10,6(9,6-10,9)^{* * *}$ & $13,2(11-15)^{*}$ & p 0,0060 \\
\hline Ferritina materna $(\mathrm{ng} / \mathrm{mL})$ & $27,3(20-36,5)$ & $7,8(7,4-12,2)^{* *}$ & $92,5(21,9-343,1)^{* *}$ & p 0,0026 \\
\hline Presión arterial sistólica (mmHg) & $113,5(110-135)^{*}$ & $116,5(105-120)^{*} *$ & $166,5(150-200)^{* *}$ & p 0,0047 \\
\hline Presión arterial diastolica $(\mathrm{mmHg})$ & $75,0(70-83)^{*}$ & $72,0(70-81)^{* *}$ & $107(94-120)^{* * *}$ & p 0,0036 \\
\hline
\end{tabular}

Los datos se muestran como medianas y sus rangos, análisis estadístico con Kruskal Wallis, desde ${ }^{*} p<0.05 y{ }^{* *} p<0.01$ 
diferencial de las proteínas de la vellosidad placentaria, como las detectadas entre $95 \mathrm{kDa}$ y $130 \mathrm{kDa}$ y entre $43 \mathrm{kDa}$ y $55 \mathrm{kDa}$, las cuales no se observaron en las mujeres con anemia. Además hubo dos bandas de proteínas que fueron visibles sólo en el grupo de mujeres con $\mathrm{PE}$, una de $43 \mathrm{kDa}$ y otra de $34 \mathrm{kDa}$. La intensidad en la tinción de las bandas fue mayor en algunas muestras del grupo de mujeres con PE comparado con el grupo control, mientras que la intensidad de las bandas de proteínas de algunas de las muestras de las mujeres con anemia fue menor. Estos hallazgos fueron confirmados por la electroforesis capilar (datos no mostrados).

Patrones de glicanos en proteínas de la vellosidad placentaria: Se realizó una densitometría de aquellas bandas de glicoproteínas que fueron comunes a los tres grupos evaluados para establecer los patrones de glicosilación diferenciales entre los grupos. En los diferentes ensayos fue posible detectar una banda de proteínas de baja glicosilación, de aproximadamente 67KDa de expresión constante, la cual se utilizó como control de carga y para la normalización de los resultados de los patrones de glicanos. Se hicieron análisis comparativos de la intensidad de las bandas por peso molecular y posteriormente una comparación con el análisis consolidado de la intensidad de todas las bandas de una misma muestra.

El patrón galactosa $\beta 1-4 \mathrm{~N}$-acetil glucosamina detectado por la lectina DSA se encontró en proteínas de placenta de tercer trimestre con pesos moleculares entre 35 kDa y 170 $\mathrm{kDa}$. Para el análisis se tuvieron en cuenta las bandas de glicoproteínas ubicadas: entre 35kDa-55kDa, entre $55 \mathrm{kDa}-72$ kDa, entre 72k Da-95 kDa, en 95 kDa, entre 95-130 kDa y cercana a $170 \mathrm{kDa}$. Al hacer la comparación de la glicosilación de las bandas por cada peso molecular (figura 2, A), la expresión de este patrón de glicosilación era mayor en el grupo de las mujeres con PE en las proteínas ubicadas entre $95 \mathrm{kDa}$ y $130 \mathrm{kDa}(p=0.0268)$ y en las proteínas ubicadas en $95 \mathrm{kDa}$ $(p=0,03)$, pero no en los otros pesos moleculares. Al consolidar todos los datos se observó una mayor expresión $(p<0.0001)$ de galactosa $\beta 1-4 \mathrm{~N}$-acetil glucosamina en glicoproteínas de las placentas de las mujeres con PE comparado con los otros dos grupos (figura 2, B).

La expresión del ácido siálico en posición a 2-6, detec-

FIGURA 2

Expresión del patrón galactosa $\beta 1-4 \mathrm{~N}$-acetil glucosamina en glicoproteínas de placenta de tercer trimestre
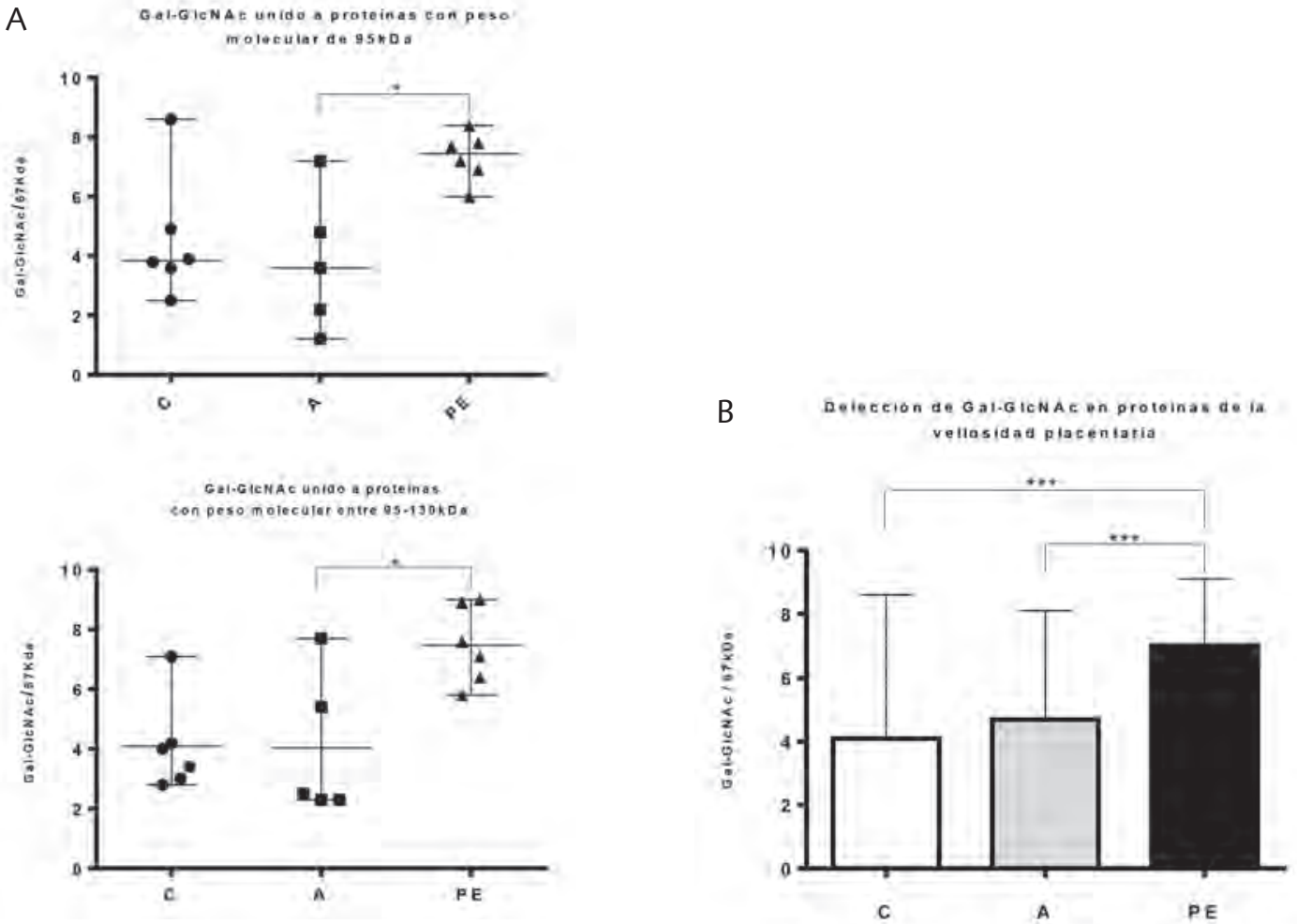

A. Expresión del patrón galactosa $31-4 \mathrm{~N}$-acetil glucosamina en las glicoproteínas con pesos moleculares en $95 \mathrm{kDa}$ y entre 95 y $130 \mathrm{KDa}$, en los grupos control (C), anemia ferropénica (A) y preeclamsia grave (PE). Los datos se muestran como medianas y sus rangos, análisis estadístico con Kruskal Wallis, desde ${ }^{*} \mathrm{p}<0.05$. 
tado por la lectina SNA, se encontró en proteínas de placenta con pesos moleculares entre 43 kDa hasta 170 kDa. Para el análisis se tuvieron en cuenta las bandas de glicoproteínas ubicadas en los rangos entre: 43 kDa-55 kDa, 55 kDa-72 kDa, 72 kDa-95 kDa, en 95 kDa y cerca de 170 kDa. Al comparar la glicosilación en las bandas por cada peso molecular, no se observó diferencia en la expresión de este patrón en los grupos (datos no mostrados). Al consolidar los datos tampoco hubo diferencia significativa en la expresión de ácido siálico en posición 2-6 en las glicoproteínas de la placenta entre los grupos (datos no mostrados).

La expresión del patrón de glicosilación manosa terminal detectado por la lectina GNA, se encontró en glicoproteínas de placenta de tercer trimestre con pesos moleculares desde 26 kDa hasta 170 kDa. Para el análisis se tuvieron en cuenta las bandas de glicoproteínas ubicadas en rangos entre: $26 \mathrm{kDa}$ y 35 kDa, 43 kDa y 55 kDa, 55 kDa y 72 kDa, 72 kDa y 95 kDa, 95 kDa y 130 kDa y entre 130 kDa y 170 kDa. Al hacer la comparación de la glicosilación de las bandas de proteínas por cada peso molecular, se observó que fue significativamente mayor en el grupo de mujeres con PE en los diferentes pesos moleculares, con $\mathrm{p}=0,0271$ para la banda ubicada entre 130 kDa y 170 kDa, $p=0,0194$ para la banda ubicada entre 72 kDa y $95 \mathrm{kDa}$ y $p=0,0037$ para la banda entre $42 \mathrm{kDa}$ y $55 \mathrm{kDa}$ (figura $3 \mathrm{~A})$. Al consolidar todos los datos se observó una diferencia significativa ( $p<0.0001)$ en la expresión de manosa terminal en diferentes posiciones (Mana1-3Man, Mana1-6Man, Mana12Man), en las mujeres con PE comparado con los otros dos grupos (figura 3, B).

La expresión de ácido siálico $\alpha 2-3$, detectado por la lectina MAA, se encontró en proteínas de placenta desde 55 kDa hasta $130 \mathrm{kDa}$. Para el análisis se tuvieron en cuenta las bandas de glicoproteínas ubicadas entre: 55 kDa y 72 kDa, entre 72 kDa y 95 kDa, en 95 kDa, entre 95 kDa y 130 kDa y en 130 kDa. Al hacer la comparación de la glicosilación por los diferentes pesos moleculares (figura 4, A), se observó que fue mayor en el grupo de las mujeres con PE para la banda de proteínas ubicada en $130 \mathrm{kDa}$. Al consolidar todos los datos, se observó una diferencia significativa $(p<0.0001)$ en la expresión de ácido sialico a2-3 en las mujeres con PE comparado con el grupo control (figura 4, B).

Diferentes ensayos se realizaron usando la lectina PNA, que detecta el núcleo disacárido Gal 1-3 GalNAc, pero en ninguno se detectó el glicano de interés. Se realizaron diferentes comprobaciones con el fin de darle validez al resultado (datos no mostrados).

\section{DISCUSIÓN}

Alteraciones fisiopatológicas como las descritas, tanto para la anemia gestacional como para la preeclampsia, se han relacionado con cambios en la placenta. La anemia genera una hipoxia preplacentaria debido a la baja tensión de oxígeno en la sangre materna (39), lo cual incrementa la angiogénesis y causa un aumento en el número de capilares, situación que en principio aumentaría la transferencia de oxígeno y nutrientes al feto. Si esta respuesta adaptativa persiste durante la gestación, estos eventos alterarían la estructura histológica de la placenta (40). De otro lado, la preeclampsia y una de sus manifestaciones más severas, la preeclampsia grave de inicio temprano, causa hipoxia utero-placentaria debido al flujo limitado de sangre por una ineficiente invasión trofoblástica a las arterias espirales, incremento en la resistencia vascular en el lecho placentario, acompañado de la liberación de sustancias vasoactivas, producción de especies reactivas del oxígeno, producción de citoquinas proinflamatorias y disfunción endotelial (41).

\section{FIGURA 3}

Expresión del patrón de glicosilación manosa terminal.

A

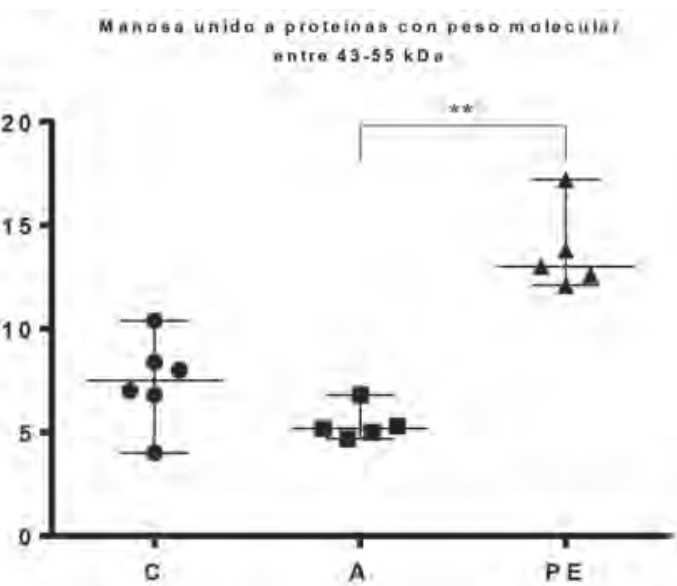

B

Detección manosa terminal unida a proteinas de vellosidad placentaria

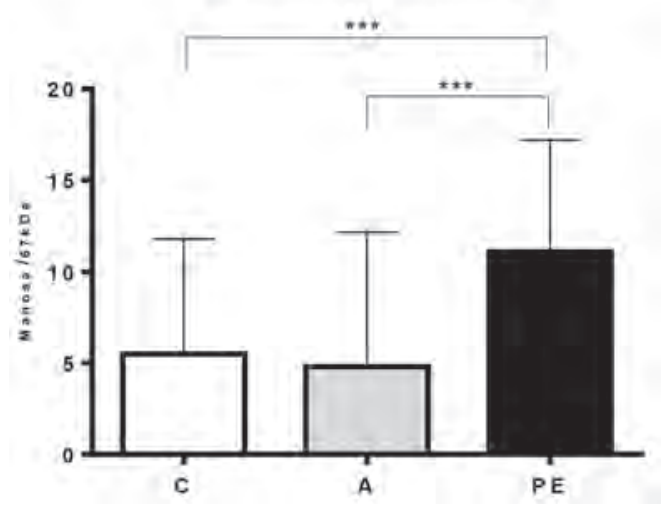

A. Expresión del patrón manosa terminal en las glicoproteínas con pesos moleculares en 45kDa y 55KDa en los grupos control (C), anemia ferropénica (A) y preeclamsia grave (PE). Los datos se muestran como medianas y sus rangos, análisis estadístico con Kruskal Wallis, desde ${ }^{* *} \mathrm{p}<0.05$.

B. Expresión del patrón de glicosilación manosa terminal de las glicoproteínas de la placenta de tercer trimestre de los tres grupos de mujeres, control (C), anemia ferropénica (A), preeclampsia grave (PE). Los datos se muestran como medianas y sus rangos, análisis estadístico con Kruskal Wallis, desde ${ }^{* * *} \mathrm{p}<0.0001$. 
Aunque a los glicanos unidos a las proteínas se les ha otorgado un papel central en el metabolismo, en la biología de la matriz extracelular y en la interacción célula-célula, las diferencias en la glicosilación en placenta de mujeres normales y de mujeres con complicaciones de la gestación ha sido escasamente explorada. Una de las primeras descripciones de los glicanos de la placenta la hicieron Arkwright y cols, a partir de placentas a término obtenidas de mujeres con gestaciones sanas; determinaron que $92 \%$ de los glicanos de la placenta son $\mathrm{N}$-glicanos, mientras los $\mathrm{O}$-glicanos sólo representan $8 \%$. Los $\mathrm{N}$-glicanos presentes en la placenta son en su mayoría de estructura química ramificada o complejos (95\%) y sólo 5\% son altamente manosilados. Además, que los oligosacáridos complejos son en su mayoría sializados (72\%) y que con el aumento de las semanas de gestación se da una reducción en la presencia de fucosa en los carbohidratos complejos (42). En este estudio, realizamos una aproximación a la caracterización de los patrones de glicosilación de la vellosidad placentaria de mujeres con alteraciones de la gestación y de mujeres sanas, usando para esto lectinas que se unen a glicotopes terminales específicos.

En las glicoproteínas de la placenta en el grupo con PE, los diferentes ensayos permitieron determinar la sobreexpresión de los patrones Gal-GlcNAc, manosa terminal y ácido sialico $\alpha 2-3$, detectados con las lectinas DSA, GNA y MAA, respectivamente, mientras que en el grupo de mujeres con anemia, no hubo cambios respecto al grupo control.

Si se tienen en cuenta los resultados obtenidos de proteínas glicosiladas que unen específicamente a la lectina GNA entre $95 \mathrm{KDa}$ y $130 \mathrm{KDa}$ en el grupo de mujeres con PE y no en los otros dos y de proteínas de peso molecular cercano a $95 \mathrm{KDa}$ y $55 \mathrm{KDa}$, que unen específicamente la lectina SNA en las mujeres sanas y con anemia ferropenica, pero no en las
$P E$, se confirma la diferencia encontrada en los patrones de glicosilación, que daría cuenta de cambios postraduccionales inducidos probablemente por la condición de estrés a la que se encuentra sometida la vellosidad placentaria durante la $P E$. Esta condición de estrés se relaciona con la activación de la vía del NF-kB, el factor inducible por la hipoxia (HIF1 $\alpha$ ), entre otros, que alteran la estructura de la vellosidad y generan mayor liberación de micropartículas de STB a la circulación sistémica (43).

Llama la atención que en el grupo de mujeres con anemia ferropénica no se encontraran diferencias con el grupo control. Como posibles explicaciones a éste fenómeno podrían considerarse: a) Que la placenta y el feto pudieran haber estado sometidos por poco tiempo a los efectos adversos de la deficiencia de hierro y quizás los eventos determinantes para producir cambios en la expresión o función de las proteínas estarían modulados por la cronicidad del déficit de hierro. Zhang y cols. concluyeron que sólo la anemia en las primeras semanas de la gestación presentaba una asociación significativa con la aparición de parto pretérmino, mientras que la anemia ocurrida en el último trimestre del embarazo mostró una reducción en el riesgo de complicaciones, especialmente en parto pretérmino (44). b) La anemia materna pudo activar mecanismos compensadores de la captación de hierro por la placenta para mantener el aporte de hierro a la placenta misma y al feto y evitar cambios en el funcionamiento de proteínas y en los resultados como BPN, parto pretérmino o inclusive anemia fetal.

Estudios utilizando en modelos murinos han revelado un aumento en el flujo de hierro al feto derivado de una regulación positiva en la expresión de proteínas relacionadas con el transporte de este nutriente, como el TfR1 y el DMT1 en ratas sometidas a diferentes grados de deficiencia de hierro

\section{FIGURA 4}

Expresión del patrón ácido siálico a2-3 en las glicoproteínas de placenta de tercer trimestre

A

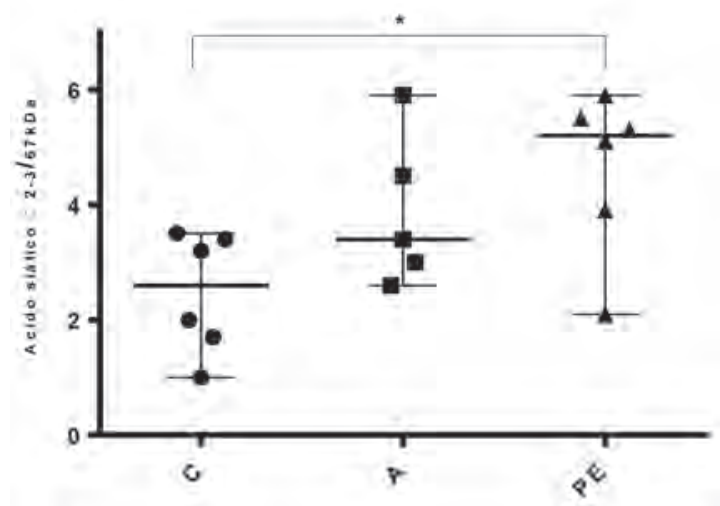

B

Detección Ácido Sialico $2-3$ unido a proteinas de la vellosidad placentaria

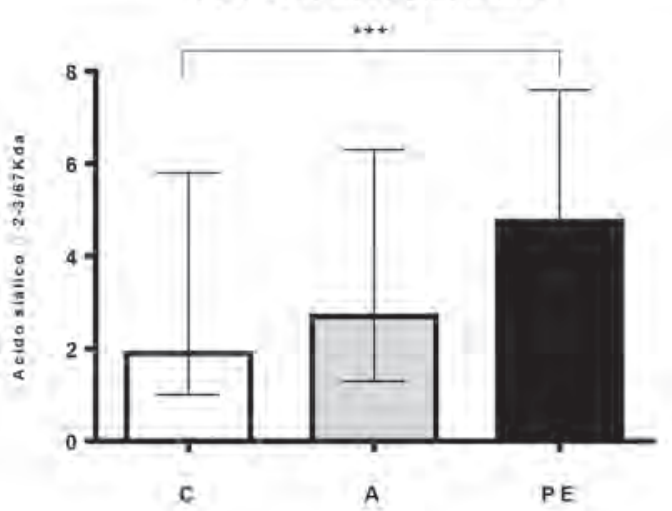

A. expresión de siálico a2-3 en las glicoproteínas con pesos moleculares en 130KDa en los tres grupos de mujeres, control (C), anemia ferropénica (A), preeclampsia grave (PE). Los datos se muestran como medianas y sus rangos, análisis estadístico con Kruskal Wallis, $d{ }^{*} p<0,05$.

B. expresión del patrón siálico a2-3 de las glicoproteínas de la placenta de los tres grupos de mujeres, control (C), anemia ferropénica (A), preeclampsia grave (PE). Los datos se muestran como medianas y sus rangos, análisis estadístico con Kruskal Wallis, $d^{* * *} p<0,0001$. 
(45). Esta respuesta ha sido encontrada en mujeres con reservas depletadas de este mineral, en las cuales se observó un aumento en la expresión del TfR1 (28). c) Las concentraciones de hemoglobina encontradas $(9,6-10,9 \mathrm{~g} / \mathrm{dL})$ en el grupo de mujeres con anemia ferropénica podrían considerarse adecuadas para el crecimiento fetal y para la expresión y función de las proteínas de la placenta. Steer y su grupo encontraron que la menor incidencia de BPN y de parto pretermino se presentó cuando la hemoglobina materna se encontraba entre 9,5 - 10,5 $\mathrm{g} / \mathrm{dL}$, valores que son considerados como anemia de acuerdo con la propuesta de la OMS (46).

Las diferencias encontradas en el patrón de glicosilación en el grupo de mujeres con PE pueden tener implicaciones en una variedad de características de las proteínas, lo que a su vez puede afectar su funcionalidad. La unión de residuos de monosacáridos terminales en las cadenas de oligosacáridos de manera diferencial en el grupo con PE, puede contribuir a cambios en la masa, en la carga y en la estructura terciaria de las proteínas por efectos estéricos en la molécula. Estos cambios podrían modificar la exportación a la membrana celular, la afinidad por sus ligandos o la actividad de las proteínas de la vellosidad trofoblástica (47), eventos que podrían afectar su funcionamiento en la captación de nutrientes hacía la placenta y afectar el estado nutricional fetal.

La presencia del patrón de glicosilación ácido siálico a2-3 detectado por la lectina MAA y ácido sialico en posición $\alpha 2-6$ en las glicoproteínas de la vellosidad trofoblástica y la sobreexpresión en las mujeres con PE del patrón ácido siálico $\alpha 2-3$, son importantes debido a las implicaciones que tiene éste residuo de carbohidratos en la respuesta inmune y en la tolerancia al feto (48). Arkwright y cols, demostraron que las glicoproteínas del trofoblasto son en su mayoría sializadas, esto genera una superficie celular inerte que inhibe la interacción y activación celular, buscando posiblemente una menor activación del sistema inmune materno (42). Por su parte Whyte y Loke, trataron células con neuraminidasa y observaron un aumento en la citolisis del trofoblasto por actividad de leucocitos alogénicos (49), lo cual sustenta la posible acción fisiológica de los ácidos sialicos terminales en el glucocalix del trofoblasto. Consideramos que el aumento en la sialización a2-3 de las glicoproteínas de placenta de tercer trimestre encontrada en nuestra investigación en el grupo de mujeres con PE, podría ser explicada por la respuesta inflamatoria sistémica y local que ocurre en la enfermedad (50) y que modifica el patrón de glicosilación de estas proteínas. Una explicación al aumento en la sialización de las proteínas de mujeres con PE, contemplaría la posibilidad de un aumento en la estabilidad de la proteína conferida por la adición de este residuo de carbohidrato. Un ejemplo de lo anterior son los experimentos de Rutledge y cols, quienes transfectaron células TRVb con TfR1 al que se le había mutado la Thr 104 por Gly, Asn, Asp, Glu, Lys, o Met. Las mediciones del TfR1 en el medio (TfR1 soluble), mostraron que la mutación con amino ácidos cargados y de grandes cadenas laterales inhibía significativamente el clivaje de la proteína, mientras que la eliminación del ácido siálico lo aumentó. Cuando la glicosilación fue defectuosa (truncada o carente de la O-glicosilación), el clivaje aumentó. Los autores concluyeron que el core GalNAc no protege el receptor contra el clivaje, mientras que la presencia de ácido sialico si lo hace. Esto sugiere que el clivaje pudiera ser regulado por la remoción parcial o total de este monosacárido (51).

Se podría considerar que el aumento general en los patrones de glicosilación encontrado en las proteínas de la placenta de mujeres con $\mathrm{PE}$, estaría relacionado con un mecanismo que busca mejorar la estabilidad de las proteínas. Se ha visto un incremento en la temperatura de transición, entendida como la temperatura a la cual una proteína tiene estabilidad cero, en la medida que incrementa el grado de glicosilación. En promedio por cada glicano adicional se incrementa la temperatura de transición entre 0,6 y $0,9^{\circ} \mathrm{C}(47)$.

Una explicación a estos cambios particulares en el patrón de glicosilación en las mujeres con PE no ha sido dada, pero algunas consideraciones pueden hacerse. La patogénesis de la $P E$, se ha relacionado con un aumento de la resistencia en las arterias espirales por una pérdida de balance entre sustancias vasocontrictoras y vasodilatadoras, debido a una invasión trofoblastica incompleta lo que conduce a un marcado ambiente hipóxico (13). El aumento del factor de transcripción HIF1a podría estar relacionado con los cambios en el perfil de glicosilación de las proteínas de placenta del grupo de mujeres con PE. Trabajos previos en nuestro grupo determinaron un aumento significativo en el antígeno Lewisy en la placa basal decidual de mujeres con PE. Como el antígeno Lewisy está implicado con procesos angiogénicos, el hallazgo fue interpretado como un mecanismo compensatorio encaminado a buscar la homeostasia del oxígeno en el tejido mal perfundido en las mujeres con PE. Un aumento en la expresión de este antígeno en células de CTB también había sido descrita por Minas en 2007 en mujeres con PE (52). Estudios recientes han demostrado un aumento en el estrés del retículo endoplasmático (RE) en mujeres con PE y en mujeres normotensas que desarrollaron RCIU (53).

Para evaluar si los mecanismos postraduccionales asociados a la glicosilación pueden tener un papel en la exportación de glicoproteínas a la membrana del STB, como es el caso de TfR1 y por lo tanto en la regulación del flujo de hierro materno fetal, estudios posteriores deberán enfocarse a caracterizar y comparar el perfil de glicosilación de esta proteína en mujeres gestantes sanas, con A y PE; además, parece importante conocer cómo es el flujo de hierro en la interface materno-fetal de la mujer con PE, por lo tanto se espera determinar la expresión del TfR1 y confirmar si en mujeres con PE de inicio temprano, se presenta una expresión a la baja de esta proteína, como fue reportado previamente para mujeres con PE.

\section{RESUMEN}

Introducción: Las glicoproteínas de la membrana del sincitiotrofoblasto (STB) se encuentran en contacto con la sangre materna, por lo que pueden participar en la comunicación en la interface materno-fetal. Objetivo: caracterizar patrones de glicanos de la vellosidad trofoblástica de mujeres sanas, anémicas por deficiencia de hierro y preeclámpticas graves de inicio temprano. Materiales y métodos: se obtuvieron extractos proteínicos de vellosidad placentaria de tercer trimestre y se determinó la expresión de patrones de glicanos, usando lectinas. Para la comparación de los grupos se utilizó la prueba de Kruskal-Wallis. Resultados: Se encontró una sobreexpresión en los patrones de glicosilación Gal-GIcNAc, manosa y ácido siálico $\alpha 2-3$ en el grupo con preeclampsia. Conclusiones: El aumento en los patrones Gal-GIcNAc, alta manosilación y ácido siálico $\alpha 2-3$, en proteínas de vellosidad placentaria en los pesos moleculares encontrados, pudiera explicar cambios en la expresión de proteínas de membrana del STB.

Palabras clave: Anemia gestacional, preclámpsia grave, hierro, receptor 1 de transferrina, glicosilación.

Agradecimientos: Los autores agradecen a la Estrategia de Sostenibilidad 2014-2015 de la Universidad de Antioquia 
y a las Dras, Angela Patricia Cadavid Directora del Grupo Reproducción y Claudia María Velásquez Directora del GIANHEND-UdeA. Al Dr Juan Guillermo Maldonado por la revisión crítica de este artículo.

\section{BIBLIOGRAFÍA}

1. Kaiser L, Allen LH, American Dietetic Association. Position of the American Dietetic Association: nutrition and lifestyle for a healthy pregnancy outcome. J Am Diet Assoc. 2008;108(3):553-61.

2. Milman N. Prepartum anaemia: prevention and treatment. Ann Hematol. 2008;87(12):949-59.

3. McCann JC, Ames BN. An overview of evidence for a causal relation between iron deficiency during development and deficits in cognitive or behavioral function. Am J Clin Nutr. 2007;85(4):931-45.

4. de Benoist B, McLean E, Egli I, Cogswell M. Worldwide prevalence of anaemia 1993-2005 WHO Global Database on Anaemia. Consultado 2013/08/04. Disponible en http://www.who.int/nutrition/publications/micronutrients/anaemia_iron_deficiency/9789241596657/en/.

5. Kalaivani K. Prevalence \& consequences of anaemia in pregnancy. Indian J Med Res. 2009;130(5):627-33.

6. Belkacemi L, Nelson DM, Desai M, Ross MG. Maternal undernutrition influences placental-fetal development. Biol Reprod. 2010;83(3):325-31.

7. Muthayya S. Maternal nutrition \& low birth weight - what is really important? Indian J Med Res. 2009;130(5):600-8.

8. Zhou LM, Yang WW, Hua JZ, Deng CQ, Tao X, Stoltzfus $R J$. Relation of hemoglobin measured at different times in pregnancy to preterm birth and low birth weight in Shanghai, China. Am J Epidemiol. 1998;148(10):998-1006.

9. Ministerio de la Protección Social, Instituto Colombiano de Bienestar Familiar, Instituto Nacional de Salud, Profamilia, Departamento Administrativo Nacional de Estadistica, Instituto Colombiano del Deporte y otros. Encuesta Nacional de la Situación Nutricional en Colombia 2010 ENSIN. Primera edición ed. Bogotá; 2011.

10. Restrepo $M S L$, Mancilla $L L P$, Parra $S B E$, Manjarrés $C$ LM, Zapata L NJ, Restrepo Ochoa PA, et al. Evaluación del estado nutricional de mujeres gestantes que participaron de un programa de alimentación y nutrición. Rev Chil Nutr. 2010;37:18-30.

11. LaMarca BD, Gilbert J, Granger JP. Recent progress toward the understanding of the pathophysiology of hypertension during preeclampsia. Hypertension. 2008;51(4):982-8.

12. Guller S. Role of the syncytium in placenta-mediated complications of preeclampsia. Thromb Res. 2009;124(4):38992.

13. Kanasaki K, Kalluri R. The biology of preeclampsia. Kidney Int. 2009;76(8):831-7.

14. Serdar Z, Gür E, Develioğlu O. Serum iron and copper status and oxidative stress in severe and mild preeclampsia. Cell Biochem Funct. 2006;24(3):209-15.

15. Toldi G, Stenczer B, Molvarec A, Takáts Z, Beko G, Rigó $J$, et al. Hepcidin concentrations and iron homeostasis in preeclampsia. Clin Chem Lab Med. 2010;48(10):1423-6.

16. Siddiqui IA, Jaleel $A$, Kadri HM, Saeed WA, Tamimi W. Iron status parameters in preeclamptic women. Arch Gynecol Obstet. 2011;284(3):587-91.

17. ACOG. Practice Bulletin. Diagnosis and management of preeclampsia and eclampsia. Obstet Gynecol. 2002;99(1):15967.

18. Espinoza J, Romero $R$, Nien JK, Gomez $R$, Kusanovic JP,
Gonçalves $L F$, et al. Identification of patients at risk for early onset and/or severe preeclampsia with the use of uterine artery Doppler velocimetry and placental growth factor. Am J Obstet Gynecol. 2007;196(4):326.e1-13.

19. López-Jaramillo $P$, Silva $F$, Camacho PA, Pradilla $L P$, García $R$, Rueda-Clausen $C$, et al. Síndrome metabólico y preeclampsia: los aportes realizados por el Instituto de Investigaciones de la Fundación Cardiovascular de Colombia. Rev Colomb Cardiol. 2006;13:73-8.

20. Soares MJ, Chakraborty D, Renaud SJ, Kubota K, Bu P, Konno $T$, et al. Regulatory pathways controlling the endovascular invasive trophoblast cell lineage. J Reprod Dev. 2012;58(3):283-7.

21. Sölder E, Rohr I, Kremser C, Hutzler P, Debbage PL. Imaging of placental transport mechanisms: a review. Eur J Obstet Gynecol Reprod Biol. 2009;144 Suppl 1:S114-20.

22. Huppertz B. The anatomy of the normal placenta. J Clin Pathol. 2008;61(12):1296-302.

23. Handwerger S. New insights into the regulation of human cytotrophoblast cell differentiation. Mol Cell Endocrinol. 2010;323(1):94-104.

24. Fuchs R, Ellinger I. Endocytic and transcytotic processes in villous syncytiotrophoblast: role in nutrient transport to the human fetus. Traffic. 2004;5(10):725-38.

25. Jansson T, Myatt L, Powell TL. The role of trophoblast nutrient and ion transporters in the development of pregnancy complications and adult disease. Curr Vasc Pharmacol. 2009;7(4):521-33.

26. Gambling $L$, Danzeisen $R$, Gair $S$, Lea RG, Charania $Z$, Solanky $N$, et al. Effect of iron deficiency on placental transfer of iron and expression of iron transport proteins in vivo and in vitro. Biochem J. 2001;356(Pt 3):883-9.

27. Li YQ, Yan H, Bai B. Change in iron transporter expression in human term placenta with different maternal iron status. Eur J Obstet Gynecol Reprod Biol. 2008;140:48-54.

28. Young MF, Pressman E, Foehr ML, McNanley T, Cooper E, Guillet $R$, et al. Impact of maternal and neonatal iron status on placental transferrin receptor expression in pregnant adolescents. Placenta. 2010;31(11):1010-4.

29. Khatun $R, W u$ Y, Kanenishi K, Ueno M, Tanaka S, Hata T, et al. Immunohistochemical study of transferrin receptor expression in the placenta of pre-eclamptic pregnancy. Placenta. 2003;24(8-9):870-6.

30. Mandò C, Tabano S, Colapietro P, Pileri P, Colleoni F, Avagliano $L$, et al. Transferrin receptor gene and protein expression and localization in human IUGR and normal term placentas. Placenta. 2011;32(1):44-50.

31. Hunt RC, Riegler $R$, Davis AA. Changes in glycosylation alter the affinity of the human transferrin receptor for its ligand. J Biol Chem. 1989;264(16):9643-8.

32. Getsios S, MacCalman CD. Cadherin-11 modulates the terminal differentiation and fusion of human trophoblastic cells in vitro. Dev Biol. 2003;257(1):41-54.

33. Chen CP, Aplin JD. Placental extracellular matrix: gene expression, deposition by placental fibroblasts and the effect of oxygen. Placenta. 2003;24(4):316-25.

34. Purcell SH, Moley $\mathrm{KH}$. Glucose transporters in gametes and preimplantation embryos. Trends Endocrinol Metab. 2009;20(10):483-9.

35. Atalah E, Castillo C, Castro R, Aldea A. Propuesta de un nuevo estandar de evaluación nutricional en embarazadas. Rev Med Chil. 1997;125(12):1429-36.

36. ACOG. Hypertension in pregnancy. Report of the American College of Obstetricians and Gynecologists' Task 
Force on Hypertension in Pregnancy. Obstet Gynecol. 2013;122(5):1122-31.

37. Cunningham F. Maternal Physiology. En: Cunningham F, Leveno K, Bloom S, Hauth J, Gilstrap III L, Wenstrom K. Williams Obstetrics. 22ed. New York. McGrawHill;2005,131.

38. Kliman HJ, Nestler JE, Sermasi E, Sanger JM, Strauss JF. Purification, characterization, and in vitro differentiation of cytotrophoblasts from human term placentae. Endocrinology. 1986;118(4):1567-82.

39. Soares MJ, Hunt JS. Placenta and trophoblast: methods and protocols: overview II. Methods Mol Med. 2006;122:3-7.

40. Kadyrov M, Kosanke G, Kingdom J, Kaufmann P. Increased fetoplacental angiogenesis during first trimester in anaemic women. Lancet. 1998;352(9142):1747-9.

41. Zamudio S. Hipoxia and the placenta. En: The Placenta: from development to disease. Kay $H$, Wang $N$, Wang $Y$. Wiley-Blackwell. 2011. Oxford UK. doi: 10.1002/978 1444393927.ch6.

42. Arkwright PD, Redman CW, Williams PJ, Dwek RA, Rademacher TW. Syncytiotrophoblast membrane protein glycosylation patterns in normal human pregnancy and changes with gestational age and parturition. Placenta. 1991;12(6):637-51.

43. Redman CW, Sargent IL. Microparticles and immunomodulation in pregnancy and pre-eclampsia. J Reprod Immunol. 2007;76(1-2):61-7.

44. Zhang Q, Ananth CV, Li Z, Smulian JC. Maternal anaemia and preterm birth: a prospective cohort study. Int J Epidemiol. 2009;38(5):1380-9.

45. Gambling L, Czopek A, Andersen HS, Holtrop G, Srai SK, Krejpcio Z, et al. Fetal iron status regulates maternal iron metabolism during pregnancy in the rat. Am J Physiol Regul Integr Comp Physiol. 2009;296:R1063-70.

46. Steer PJ. Maternal hemoglobin concentration and birth weight. Am J Clin Nutr. 2000;71(5 Suppl):1285S-7S.

47. Shental-Bechor D, Levy Y. Folding of glycoproteins: toward understanding the biophysics of the glycosylation code. Curr Opin Struct Biol. 2009;19(5):524-33.

48. Toscano M, Ilarregui J, Bianco G. Interacción entre proteínas y glicanos en la regulación fisiológica de células T. Medicina. 2006;66:357-62.

49. Whyte A, Loke YW. Increased sialylation of surface glycopeptides of human trophoblast compared with fetal cells from the same conceptus. J Exp Med. 1978;148(4):108792.

50. Bueno-Sánchez JC, Agudelo-Jaramillo B, Escobar-Aguilar LF, Lopera A, Cadavid-Jaramillo AP, Chaouat G, et al. Cytokine production by non-stimulated peripheral blood NK cells and lymphocytes in early-onset severe pre-eclampsia without HELLP. J Reprod Immunol. 2013;97(2):223-31.

51. Rutledge EA, Enns CA. Cleavage of the transferrin receptor is influenced by the composition of the O-linked carbohydrate at position 104. J Cell Physiol. 1996;168(2):284-93.

52. Minas V, Mylonas I, SchiessI B, Mayr D, Schulze S, Friese $K$, et al. Expression of the blood-group-related antigens Sialyl Lewis a, Sialyl Lewis $x$ and Lewis $y$ in term placentas of normal, preeclampsia, IUGR- and HELLP-complicated pregnancies. Histochem Cell Biol. 2007;128(1):55-63.

53. Lian IA, Løset $M$, Mundal SB, Fenstad $M H$, Johnson MP, Eide $I P$, et al. Increased endoplasmic reticulum stress in decidual tissue from pregnancies complicated by fetal growth restriction with and without pre-eclampsia. Placenta. 2011;32(11):823-9. 